\title{
Programas Preventivos Brasileiros: Quem Faz e como É Feita a Prevenção em Saúde Mental?
}

\author{
Samia Abren - Universidade de Brasília, Brasília, Brasil \\ Ana Aparecida Vilela Miranda - Universidade de Brasilia, Brasilia, Brasil \\ Sheila Giardini Murta - Universidade de Brasilia, Brasília, Brasil
}

\begin{abstract}
Resumo
O objetivo do estudo é identificar os centros de pesquisa brasileiros que desenvolvem intervenções preventivas sistematicamente avaliadas e descrever os estudos de avaliação de programas realizados no Brasil. Para tanto, foi realizada uma revisão sistemática da literatura nacional nas bases de dados Scielo e Pepsic que, com as seguintes palavras-chave "prevenção", "atenção primária", "atenção básica" e "promoção de saúde", resultaram em 25 artigos que descrevem 42 intervenções. Os resultados indicaram uma predominância de intervenções longas, com amostras pequenas, realizadas semanalmente, para promoção de competências em crianças e adolescentes em ambientes educacionais. Majoritariamente, os estudos omitem avaliações de seguimento e tampouco discutem as implicações dos resultados para as políticas públicas. Os pesquisadores são, em sua maioria, psicólogos, afiliados a universidades públicas da região sudeste. Conclui-se que a formação de redes de pesquisa e a ampliação de intercâmbio entre a academia e as políticas públicas podem fomentar o avanço da área no país.

Palavras-chave: prevenção, saúde mental, políticas públicas, avaliação de programas, intervenção precoce
\end{abstract}

Brazilian Preventive Programs: Who Does Them and How Does Prevention on Mental Health is Developed?

\begin{abstract}
The goal of this study is to identify the Brazilian research centers where systematic preventive interventions are developed and describe the national program evaluation studies. For that, we conducted a systematic review of the national literature on two databases (Scielo and Pepsic), through the keywords "prevention", "basic care", "primary care" and " health promotion ", which resulted in 25 articles, describing 42 interventions. The results indicated a predominance of extensive interventions, with small samples, performed weekly, for promoting skills in children and adolescents in educational settings. Mostly, studies did not include follow-up assessments, neither discussed the implications of the findings for public policy. The researchers are mostly psychologists, affiliated to public universities in the Southeast region. It is concluded that the improvement of research networks and the expansion of exchanges between researches and public policy can foster the advancement of the area in the country. Keywords: prevention, mental health, public policies, program evaluation, early intervention
\end{abstract}

Programas Preventivos Brasileños: ¿Quién Hace y cómo Es Hecha Prevención en Salud Mental?

\begin{abstract}
Resumen
El objetivo de este estudio fue identificar los centros de investigación brasileños que desarrollan intervenciones preventivas sistemáticamente evaluadas, y también describir los estudios de evaluación de programas realizados en Brasil. Se realizó una revisión sistemática de literatura nacional en las bases de datos Scielo y Pepsic, usando como palabras clave "prevención", "atención primaria", "atención básica” y "promoción de salud”; los resultados fueron 25 artículos que describen 42 intervenciones y los mismos indicaron un predominio de intervenciones largas, con muestras pequeñas, realizadas semanalmente, para promocionar competencias en niños y adolescentes en ambientes educativos. En su mayoría, los estudios omiten evaluaciones de seguimiento y no discuten las implicaciones de los resultados en las políticas públicas. Los investigadores en su mayoría son psicólogos, afiliados a universidades públicas de la región sudeste. La formación de redes de investigación y la ampliación de intercambio entre la academia y las políticas públicas pueden fomentar el avance del área en el país.

Palabras clave: prevención, salud mental, políticas públicas, evaluación de programas, intervención precoz
\end{abstract}

Um crescimento acentuado nas pesquisas em prevenção em saúde mental tem ocorrido nas últimas décadas. Ao final da década de 50, nos EUA, George Albee (1982) conduziu estudos pioneiros acerca da prevenção às psicopatologias, os quais deram início a um fértil campo de pesquisa na América do Norte nas décadas posteriores. Argumentava-se que a incidência de psicopatologias poderia ser reduzida por meio do incremento de fatores de proteção, como autoestima, rede de apoio, habilidades de enfrentamento, práticas de socialização positivas, pertencimento e engajamento social e a redução de estressores ambientais, como as injustiças sociais e os abusos aos direitos humanos, e pessoais, a exemplo da violência e da privação de vínculos afetivos (Dalton, Elias, \& Wandersman, 2007).

A prevenção em saúde mental tem por foco o desenvolvimento saudável do indivíduo em suas diversas fases do ciclo da vida e busca reduzir, por meio de ações antecipadas, os problemas e riscos que impedem tal desenvolvimento (O'Connel, Boat, \& Warner, 2009). Nessa direção, as intervenções preventivas em ação em saúde mental buscam fortalecer os fatores de proteção 
da população-alvo, sejam eles individuais ou ambientais, e minimizar os efeitos dos fatores de risco para um desfecho negativo em saúde mental. Uma vez que esses fatores estão presentes do nível micro ao macro, os contextos propícios ao desenvolvimento de programas de prevenção são os mais diversos, como escola, família, locais de trabalho, comunidade, e organizações (Dalton, et al., 2007; Weisz, Sandler, Durlak, \& Anton, 2005). Para isso, diversos avanços ocorreram na área, incluindo o surgimento de sociedades científicas, como a Society for Prevention Research, no início dos anos 90 e, mais recentemente, a European Society for Prevention Research, em 2010; a criação de periódicos especializados, como o Prevention Science Journal, em funcionamento desde 2000; e, finalmente, a expansão de programas de pós-graduação especializados, notadamente em países do hemisfério norte (Eddy, 2011).

Ao redor do mundo, os avanços da pesquisa em prevenção têm sido corroborados por evidências da eficácia e efetividade das intervenções preventivas em saúde mental. A Organização Mundial de Saúde (World Health Organization, 2004) sumarizou os programas com resultados baseados em evidências para reduzir o risco de incidência e desenvolvimento de transtornos mentais, indicando uma lista de programas que poderiam ser disseminados em diferentes contextos e/ou inseridos em políticas públicas mundiais para a prevenção de depressão, transtornos de ansiedade, transtornos alimentares, transtornos relacionados ao uso de substâncias, transtornos psicóticos e suicídio. Entretanto, nenhum programa brasileiro foi localizado e inserido nesse documento.

Por eficácia, entende-se a identificação de efeitos positivos de um programa ou política em situações ótimas ou ideais de intervenção, enquanto a efetividade se refere aos efeitos que se encontram em situações do mundo real ou cotidianas (Flay et al. 2005). A análise desses critérios proporciona maior confiabilidade dos resultados encontrados e favorecem a sua disseminação (Flay et al., 2005; Murta, 2011; Murta 2007). Além do mais, os programas, para se mostrarem efetivos e eficazes, devem ser construídos conforme certos padrões, em que as intervenções são embasadas em teoria, com planejamento sistemático e com implementação avaliada de forma rigorosa (Murta, 2011).

No Brasil, as características dos programas preventivos em saúde mental e seus resultados são desconhecidos, como apontado por revisões anteriores (referência omitida para evitar identificação de autoria deste artigo; Benetti, Ramires, Schneider, Rodrigues, \&
Tremarin, 2007).Uma revisão sistemática sobre o estado da arte da pesquisa em prevenção em saúde mental da literatura nacional (referência omitida para evitar identificação de autoria deste artigo) constatou a escassez de revisões específicas de programas nacionais. Em acréscimo, esta revisão encontrou 4131 artigos científicos nacionais que citavam prevenção, $651 \mathrm{com}$ foco em saúde mental, mas somente 11 descreviam intervenções sistematicamente avaliadas de prevenção em saúde mental. Contudo, o estudo não analisou as características das intervenções e os resultados encontrados. Assim, a lacuna na identificação das características dos programas preventivos nacionais persiste.

$\mathrm{Na}$ literatura brasileira, percebe-se um movimento na direção de publicações que priorizem avaliações sistemáticas de intervenções em saúde mental, embora análises de evidências de eficácia e efetividade de programas preventivos nacionais sejam pouco discutidas (Canoletti \& Soares, 2004; Murta, 2007). Uma publicação pioneira de Melnik e Atallah (2011) disponibilizou um panorama das evidências atuais nessa área, nomeada "Psicologia Baseada em Evidências". Entretanto, foram abordadas, majoritariamente, revisões sistemáticas sobre os efeitos de tratamento de transtornos mentais. Foram descritas 10 revisões sistemáticas em saúde mental e somente uma delas abordou a prevenção, cujo alvo foi a produção internacional, com a análise de artigos encontrados em bases de dados internacionais.

Revisões sistemáticas da literatura que evidenciam o estado da arte da pesquisa em prevenção em saúde mental no Brasil se alinham à necessidade de difusão do conhecimento, encorajada por políticas científicas recentes. A criação de novos mecanismos e investimentos públicos e privados para a transferência de conhecimento em tecnologia e práticas vem sendo discutida, a fim de que o conhecimento produzido nas universidades possa chegar à população (Marques, 2012). Na mesma direção, o Conselho Nacional de Desenvolvimento Científico e Tecnológico (CNPq), em iniciativa inovadora no Brasil, estimula os pesquisadores a divulgarem seus resultados em mídias não científicas, bem como utilizarem uma linguagem acessível ao público leigo (Conselho Nacional de Desenvolvimento Científico e Tecnológico, 2012). Frente a essas novas demandas, a identificação dos centros de pesquisas nacionais e das características de intervenções preventivas em saúde mental torna-se atual e relevante.

Com vistas à expansão do conhecimento em prevenção em saúde mental produzido no Brasil, 
realizou-se uma revisão sistemática da literatura nacional. Os objetivos do estudo foram identificar os centros de pesquisa brasileiros que desenvolvem intervenções preventivas sistematicamente avaliadas e descrever os estudos de avaliação de programas preventivos realizados no Brasil, no que diz respeito às suas características metodológicas, conteúdo e formato da intervenção e resultados obtidos.

\section{Método}

Foi realizada uma revisão sistemática da literatura nacional, identificando artigos científicos que descreviam intervenções de prevenção primária, sistematicamente avaliadas, para desfechos negativos em saúde mental. A busca foi conduzida sem limite inicial de data para publicação. Foram incluídos artigos publicados até março de 2012.

Os artigos iniciais foram encontrados em revisão sistemática anterior (referência omitida para evitar identificação de autoria deste artigo) que utilizou as palavras-chave "prevenção", "atenção primária", "atenção básica" e "promoção de saúde", nas bases SciELO e PePSIC, até janeiro de 2012, tendo localizado 11 intervenções sistematicamente avaliadas. A partir dessas, foram identificados os autores dos estudos e realizada nova busca por artigos listados em seus currículos, disponíveis on-line na Plataforma Lattes do CNPq até março de 2012. A busca por novos artigos a partir da Plataforma Lattes foi a alternativa encontrada para localizar arquivos que preenchiam os critérios de inclusão, mas que não tinham em suas palavras-chave os descritores utilizados nesta pesquisa. Essa ampliação da busca se dá pela identificação de que vários autores brasileiros conduziram intervenções preventivas, mas nomearam as suas intervenções com foco na condição prevenida (como agressividade, estresse e outros) e não utilizaram os descritores do campo da prevenção. Quando os novos artigos encontrados indicavam autores ainda não pesquisados, o currículo Lattes desses era, igualmente, analisado. Essa busca foi finalizada quando os currículos Lattes de todos os autores que publicaram intervenções sistemáticas foram analisados. Foram incluídos artigos de pesquisadores brasileiros publicados em revistas nacionais e internacionais, escritos em português, inglês e espanhol.

\section{Procedimentos de Seleção, Critérios de Inclusão e Exclusão}

A seleção dos artigos foi realizada por um pesquisador, especialista em programas preventivos.
Inicialmente, todos os artigos científicos, listados no currículo Lattes dos pesquisadores identificados em revisão de literatura prévia (referência omitida para evitar identificação de autoria deste artigo), foram analisados, exceto por cinco artigos citados no Lattes dos pesquisadores que não puderam ser localizados e analisados por limitações de acesso ao periódico.

Para ser incluído na revisão, o artigo deveria descrever (a) uma intervenção para prevenção a um transtorno mental (desfecho final), conforme classificação do Manual de Diagnóstico e Estatística dos Transtornos Mentais (Associação de Psiquiatria Americana, 2003) ou um fator de risco para transtornos mentais (desfecho intermediário) e (b) apresentar avaliação sistemática dos seus resultados. Para ser classificada como tal, a intervenção descrita deveria apresentar os seguintes critérios (Doughty, 2005): (a) delimitação clara da(s) variável(is) avaliada(s) na intervenção, (b) análise de uma variável que indicasse um desfecho final ou intermediário em saúde mental, (c) apresentação de medidas qualitativas e/ou quantitativas específicas para mensurar a variável citada e (d) apresentação de dados resultantes em resposta ao método apresentado.

Foram excluídos os artigos que descreviam estudos de: (a) prevenção secundária ou terciária, ou seja, artigos que tratavam da prevenção a danos resultantes de um transtorno mental já diagnosticado, (b) uso de tecnologias biomédicas de intervenção, como medicamentos ou procedimentos cirúrgicos, (c) estudos que descreviam parte de tratamentos ou que mesclavam prevenção e tratamento, (d) estudos que descreviam resultados da avaliação de processo da intervenção, (e) estudos que apresentavam resultados da implantação de treinamentos para a prevenção, mas não avaliavam os resultados do treinamento para o público-alvo e (f) estudos que produziam resultados exclusivamente relacionados à promoção de saúde e não indicavam efeitos preventivos para desfechos negativos em saúde mental.

A etapa de categorização foi realizada por dois codificadores e um juiz, solicitado em caso de divergência, todos com experiência em avaliação de programas preventivos. Cada codificador preenchia uma folha de codificação com os critérios apresentados, e os resultados eram comparados por meio do cálculo de concordância entre codificadores (Kazdin, 1982). O cálculo de concordância consistiu no número de concordâncias dividido pela somatória de concordâncias e discordâncias, multiplicados por 100. Em caso de divergência entre os codificadores, o juiz era solicitado 
e discussões sucessivas levavam à definição final da categoria. O grau de concordância geral encontrado, considerando todos os critérios avaliados, foi de $92,7 \%$.

\section{Categorizacão das Características das Intervencões Preventivas}

A Tabela 1 apresenta as categorias de análise utilizadas e suas definições.

\section{Resultados}

Foram incluídos 25 artigos científicos que preenchiam os critérios de inclusão. Esses artigos descrevem 42 intervenções de prevenção primária em saúde mental, sistematicamente avaliadas. Alguns estudos apresentavam comparação entre duas ou mais intervenções ou intervenções com foco combinado (no ambiente e na pessoa), o que resultou em um número maior de intervenções do que de artigos. O primeiro artigo foi publicado em 2000 e o mais recente, em 2011. Entre 2000-2002, apenas um artigo foi publicado. Entre 2003-2005, esse número subiu para seis, alcançando, em seguida, o maior número de publicações entre 20062008, com 10 artigos, e finalizando com oito artigos publicados entre 2009-março de 2012.

Os periódicos de publicação foram prioritariamente na área de psicologia. Do total, 22 periódicos eram de psicologia, um da área de educação e sexualidade, uma revista médica e um periódico de saúde coletiva. Em relação à indicação de apoio financeiro, nove artigos (36\%) citaram o apoio de agências de fomento científicas nacionais (CAPES e CNPq) e fundações regionais de amparo à pesquisa (FAPESP, FAPEMIG) e um artigo (4\%) citou o apoio do Ministério da Saúde (Secretaria de Vigilância e Saúde). Dos nove artigos que receberam apoio, todos citaram que ele ocorreu por meio de bolsas de mestrado, doutorado ou de produtividade em pesquisa.

\section{Perfil do Pesquisador da Prevenção em Saúde Mental no Brasil}

Pesquisadores seniores e jovens pesquisadores compuseram a maior parte dos autores identificados. Ao todo, 44 pesquisadores foram autores dos 25 artigos analisados. Dentre eles, 15 autores são pesquisadores seniores, segundo critérios da CAPES, ou seja, doutores há mais de 10 anos e docente/pesquisador com produção científica relevante na última década, 12 são jovens pesquisadoras (doutores há menos de 10 anos), oito são estudantes de pós-graduação, divididos entre alunos de mestrado, doutorado e especialização, e nove são alunos de graduação. A continuidade de produção não foi distribuída homogeneamente, 14 autores apresentaram produção científica contínua em prevenção. As publicações constantes foram identificadas pela análise do currículo Lattes, referindo-se a artigos, livros, capítulos de livros e resumos expandidos em congressos, com conteúdos sobre a elaboração e replicação de intervenções, criação de manuais e instrumentos de avaliação. Entretanto, 30 autores tiveram uma única publicação de intervenção preventiva e, conforme informações disponíveis nos currículos Lattes, não apresentam outras publicações de destaque para a prevenção em saúde mental.

A filiação institucional dos pesquisadores está centralizada em universidades públicas brasileiras, como pode ser observado na Figura 1. Não foram encontradas afiliações a centro de pesquisas independentes de instituições de ensino superior. A região com o maior número de grupos de pesquisa em prevenção é o sudeste, com sete das 11 universidades identificadas. Não foram encontrados publicações ou pesquisadores afiliados às universidades do norte do Brasil.

Os pesquisadores basearam suas intervenções, em sua maioria, na abordagem comportamental. Dos 25 artigos publicados, 14 descreveram intervenções baseadas na teoria comportamental $(56 \%)$, oito na teoria cognitivo-comportamental $(32 \%)$ e os demais se dividiram em uma frequência única (4\% cada) de intervenções baseadas na logoterapia, no modelo transteórico de mudança e um artigo não citou a abordagem.

\section{Características das Intervenções de Prevenção Primária em Saúde Mental}

O delineamento utilizado foi, em mais da metade, caracterizado por intervenções pré-experimentais $(54,48 \%)$, seguidas por quase experimentais $(34,88 \%)$ e experimentais $(11,62 \%)$ (ver Tabela 2). Ao todo, foram identificadas cinco intervenções experimentas e três delas são delineamentos do tipo $\mathrm{AB}$ com o número de sujeitos variando entre dois, 26 e 34 participantes. As outras duas intervenções são baseadas no modelo de Intervenções Breves (IB), com a duração de 2-3 minutos e 20 minutos, individualmente, com 99 participantes.

Intervenções breves e individuais são exceções. As outras 41 intervenções descritas (95,34\%) foram em grupo e com duração total variando entre 11 horas a um ano letivo, semanalmente. $\mathrm{Na}$ descrição dos procedimentos, 11 intervenções não informaram a quantidade de horas totais, cinco intervenções foram realizadas durante um ano letivo e, para as demais, 19 
Tabela 1

Categorias de Análise e Definições

\begin{tabular}{ll}
\hline Categoria & Definição e/ou exemplos \\
\hline 1.1. Ano & 1. Características do estudo \\
1.2. Área de atuação dos autores & $\begin{array}{l}\text { Ano de publicação do estudo. } \\
\text { Área descrita pelo autor em seu Currículo Lattes em março de 2012. Ex.: } \\
\text { psicologia, saúde pública, educação, outros. }\end{array}$ \\
$\begin{array}{l}\text { Afiliação institucional segundo informado no currículo Lattes. } \\
\text { 1.3. Instituição dos autores }\end{array}$ & $\begin{array}{l}\text { Desfecho negativo em saúde mental que é o foco da intervenção } \\
\text { (depressão, violência, uso de álcool e drogas, etc.). }\end{array}$ \\
1.4. Condição prevenida & $\begin{array}{l}\text { Variável que define a condição prevenida (rejeição por pares, } \\
\text { comportamentos agressivos, uso de álcool etc.). }\end{array}$ \\
& $\begin{array}{l}\text { Presença ou não de apoio por órgãos de fomento em pesquisa e/ou } \\
\text { instituições financiadoras públicas ou privadas. }\end{array}$ \\
\hline 1.6. Suporte financeiro &
\end{tabular}

\section{Tipo de prevenção}

2.1. Prevenção Universal

(Muñoz, Mrazek, \& Haggerty, 1996)

2.2. Prevenção Seletiva

(Muñoz et al., 1996)

2.3. Prevenção Indicada

(Muñoz et al., 1996)
Participam todos os membros de uma população, independentemente de seus fatores de risco e sintomas iniciais.

Participam indivíduos ou subgrupos selecionados pela sua exposição a fatores de risco previamente identificados.

Participam indivíduos ou subgrupos que apresentam sintomas iniciais do problema.

\section{Delineamento e características da intervenção}

3.1. Delineamento

3.2. Contexto de aplicação

3.3. Público-alvo

3.4. Características das sessões

3.5. Condição promovida

(1) Estudos experimentais: utilizam grupo controle e experimental com distribuição aleatória dos participantes, (2) quase experimentais: apresentam grupo controle e experimental sem distribuição aleatória e (3) pré-experimentais: incluem avaliação pré e pós-teste ou somente pósteste, sem grupo de comparação (Campbell \& Stanley, 1979).

Local onde foi realizada a intervenção (escola, comunidade, hospital, empresa, universidade, etc.).

Participantes da intervenção (crianças, adolescentes, adultos, casais, professores, pais, etc.) e número de participantes.

Número de sessões e quantidade total de horas de oferta da intervenção.

O fator de proteção promovido, descrito como conteúdo da intervenção (treinamento de habilidades sociais, estratégias de enfrentamento ao estresse, habilidades parentais, etc.).

\section{Foco da intervenção}

4.1. Estratégias de intervenção (Durlak \& Wells, 1997)

(1) Centradas no ambiente, isto é, dirigidas à mudança do contexto ou de comportamentos de indivíduos que lidam diretamente com a populaçãoalvo (2) centradas na pessoa, isto é, focadas diretamente no público-alvo (3) programas combinados, que incluem intervenções centradas no ambiente e na pessoa.

\section{Avaliação dos resultados}

5.1. Descrição dos resultados

\subsection{Follow-up}

5.3. Discussão sobre políticas públicas
Resultados descritos em, ao menos, uma variável avaliada.

Presença ou não de follow-up, tempo de realização e seus resultados.

Presença ou não de discussões sobre as implicações dos resultados do estudo para as políticas públicas. 


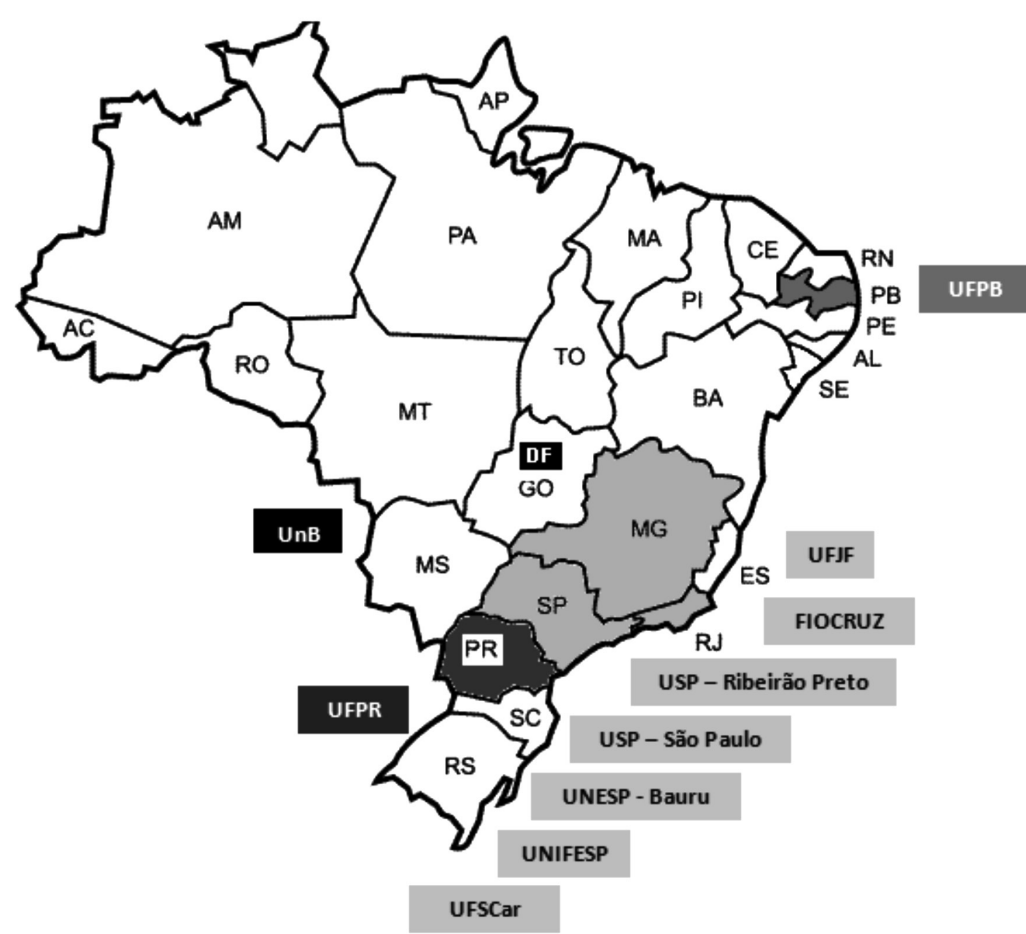

Figura 1. Mapa do vínculo institucional dos pesquisadores de prevenção primária em saúde mental.

intervenções, que descreveram as horas de contato entre pesquisadores e participantes, a média total foi de 30 horas por participante, em grupo.

As características da amostra destacaram como população-alvo as crianças e adolescente em idade escolar. Exceto por três intervenções, que focavam a prevenção do estresse ocupacional em adultos, as outras $39(92,85 \%)$ tinham como meta final a prevenção de fatores de risco à saúde mental de crianças e adolescentes. A escolha dos participantes conforme a exposição da população aos fatores de risco foi dividida entre intervenções universais e indicadas ambas com porcentagem similar de 43\%. As intervenções seletivas tiveram a menor proporção, com menos de $15 \%$ de frequência.

Predominaram as intervenções com foco combinado. Em 28 intervenções (65,11\%), o público-alvo final e pessoas relevantes ao seu contexto receberam intervenções visando à melhora ou à redução de fatores de risco no comportamento da população-alvo. As demais intervenções foram distribuídas entre intervenções focadas na pessoa $(18,6 \%)$ e no ambiente (16,27\%). Em intervenções com foco no ambiente, os que recebiam a intervenção eram pais, professores ou cuidadores da população infanto-juvenil. Não foram identificadas intervenções ambientais em mudanças organizacionais ou em políticas públicas.
Em consonância com o público-alvo, o local de aplicação majoritário foi o contexto educacional. A escola foi o local de implantação dos programas em $62,79 \%$ das intervenções, seguidos por intervenção em clínica-escola (16,27\%), ambientes comunitários $(13,95 \%)$, como ONG, centros comunitários e centros de referência em assistência social e contextos ocupacionais $(6,97 \%)$. O número de participantes apresentou grande variação, de intervenções com dois sujeitos a populações de 11.337 adolescentes. As intervenções com o maior número de sujeitos se concentraram nas intervenções ambientais de prevenção a violência, descritas em um artigo que analisou iniciativas bem-sucedidas de prevenção à violência (Gomes, Minayo, Assis, Njaine, \& Schenker, 2006). Nessas, como pode ser observado na Tabela 2 , as intervenções caracterizaram-se em comunitárias e com atividades relacionadas ao esporte, capacitação profissional e identidade cultural. As intervenções com um número reduzido de sujeitos (menos de 50) foram as intervenções longas (30 horas a um ano letivo), realizadas semanalmente e que tinham como objetivo o ensino de competências.

As condições prevenidas foram os fatores de risco à saúde mental. Nenhum artigo elencou ou avaliou a prevenção de transtornos mentais. Dentre os fatores de risco, os problemas de comportamento foram 
enunciados como foco de 61,36\% das intervenções, seguidos por intervenções de prevenção à violência $(13,64 \%)$, abuso sexual $(9,09 \%)$, abuso de álcool e drogas $(6,82 \%)$, estresse $(6,82 \%)$ e a prevenção do vazio existencial $(2,27 \%)$. A fim de mensurar tais condições prevenidas, a escolha da variável dependente avaliada não foi homogênea. Para a prevenção de problemas de comportamento, por exemplo, os estudos elencaram como variável dependente a rejeição por pares, comportamentos agressivos, comportamentos inibidos, comportamentos não assertivos, conflitos interpessoais, conflitos abertos, comportamentos socialmente aversivos e práticas parentais inadequadas. A prevenção da violência foi enunciada por meio de variáveis, como o manejo coercitivo de cuidadores de crianças em situação de abrigo, identificação de sinais de abuso e práticas parentais inadequadas (ver Tabela 2).

Em ordem decrescente de ocorrência, os conteúdos das intervenções caracterizaram-se pelo ensino de práticas parentais positivas baseadas na análise do comportamento $(17,65 \%)$, seguidas pelo ensino de habilidades sociais educativas parentais (13,73\%), resolução de problemas para crianças e adolescentes $(13,73 \%)$, habilidades sociais para diferentes faixas etárias $(13,73 \%)$ e intervenções com foco em recursos ambientais, com o desenvolvimento de atividades esportivas, lúdicas e ocupacionais $(11,76 \%)$. Os conteúdos das demais intervenções foram o ensino de estratégias de manejo de estresse $(9,8 \%)$, o manejo de dificuldades acadêmicas (3,92\%) e o ensino de habilidades de vida $(3,92 \%)$. Foram identificadas quatro intervenções com conteúdos específicos com uma única ocorrência (frequência de 1,96\% cada), uma para o ensino de efeitos do uso de álcool e outras drogas (no formato informativo) e outra para o ensino de estratégias de enfrentamento na prevenção do uso abusivo de álcool e outras drogas. As outras duas intervenções tiveram como conteúdo principal o sentido da vida e outra sobre o desenvolvimento moral (ver Tabela 2).

Quando as habilidades sociais educativas parentais, resolução de problemas e habilidades sociais gerais são agrupadas na categoria "habilidades sociais", essas passam a representar $41,19 \%$ da frequência total das condições promovidas. Esse número integra a porcentagem de estudos que não nomeiam a própria prática como preventiva, que totalizam 11 dos 25 estudos analisados (44\%). Como pode ser observado na Tabela 2, os estudos têm como foco a promoção de competências, entretanto, avaliam variáveis preventivas em seus resultados.
Os resultados dos estudos indicaram que as intervenções foram bem-sucedidas na prevenção das variáveis avaliadas. Resultados expressivos incluem o aumento do repertório de habilidades sociais infantis e a diminuição de problemas de comportamento, avaliadas como as diferentes variáveis dependentes anteriormente apresentadas (rejeição por pares, comportamentos antissociais e conflitos interpessoais). As intervenções focadas no ambiente, principalmente as que se referem ao treinamento de práticas educativas parentais, alcançaram mudanças de comportamentos nos pais/cuidadores submetidos à intervenção e em seus filhos/ crianças abrigadas. Mesmo quando as crianças não haviam recebido nenhuma intervenção, os instrumentos qualitativos e/ou quantitativos indicaram mudanças cognitivas e comportamentais nestas, o que reforça o valor de intervenções ambientais na prevenção em saúde mental (Tabela 2).

Intervenções combinadas, focadas na pessoa e no ambiente, alcançaram impactos práticos e de mudanças sociais na vida dos participantes e na dinâmica de sua comunidade. Intervenções que focavam mudanças pessoais cognitivas e mudanças sociais, como a diminuição do preconceito, fortalecimento da identidade e reinserção profissional apresentaram impactos diretos no aumento da autoestima dos participantes e na minimização do envolvimento destes em episódios de delinquência e violência (Gomes, Minayo, Assis, Njaine, \& Schenker, 2006). Entretanto, outros não avaliaram os impactos sociais de suas intervenções e enfatizaram a necessidade de avaliá-los, por meio de instrumentos validados e medidas objetivas da realidade.

Intervenções breves mostraram-se efetivas na prevenção de uso de álcool e drogas entre adolescentes (De Micheli, Fisberg, \& Formigoni, 2004), assim como intervenções longas, com duração de um semestre (Borges \& Marturano, 2003) ou um ano (Borges \& Marturano, 2010; Silvares \& Melo, 2008) mostraram-se eficazes na prevenção de problemas de comportamento na infância. Não foi indicada uma quantidade de horas mínimas ou máximas para a duração total das intervenções, visto que elas apresentavam diferenças quanto à condição prevenida, a população-alvo e o alcance em número de sujeitos da população-alvo.

A presença de follow-up e a discussão de políticas públicas quase não estão presentes nos estudos brasileiros. Apenas sete $(15,9 \%)$, das 44 intervenções avaliadas, tiveram follow-up. Dessas, seis intervenções tiveram um único follow-up que ocorreu cinco meses (1 intervenção), seis meses ( 3 intervenções) e um ano ( 2 intervenções) 
Tabela 2

Sistematização dos Programas Preventivos Brasileiros Descritos em Artigos

\begin{tabular}{|c|c|c|c|}
\hline Autor e ano & $\begin{array}{l}\text { Nível de } \\
\text { Prevenção } \\
\text { Primária }\end{array}$ & $\begin{array}{l}\text { Tipo de } \\
\text { Delineamento }\end{array}$ & $\begin{array}{l}\text { Formato da Intervenção (participantes, tipo } \\
\text { de intervenção, duração, local). }\end{array}$ \\
\hline $\begin{array}{l}\text { Aquino, Silva, } \\
\text { Figueiredo, Dourado, \& } \\
\text { Farias (2011). }\end{array}$ & Universal & Quase experimental & $\begin{array}{l}33 \text { adolescentes. Grupo semanal (duas vezes } \\
\text { por semana), } 12 \text { horas, } 15 \text { sessões. Escola. }\end{array}$ \\
\hline $\begin{array}{l}\text { Bolsoni-Silva \& } \\
\text { Marturano (2010). }\end{array}$ & Indicada & Quase experimental, & $\begin{array}{l}15 \text { pais e cuidadores. Grupo semanal, 21-28 } \\
\text { h, } 14 \text { sessões. Escola e clínica escola. }\end{array}$ \\
\hline $\begin{array}{l}\text { Bolsoni-Silva, Silveira \& } \\
\text { Marturano (2008). }\end{array}$ & Universal & $\begin{array}{l}\text { Pré-experimental, } \\
\text { follow-up. }\end{array}$ & $\begin{array}{l}14 \text { pais. Grupo semanal (duas vezes por } \\
\text { semana), } 40 \text { horas. Clínica-escola. }\end{array}$ \\
\hline $\begin{array}{l}\text { Bolsoni-Silva,Silveira \& } \\
\text { Ribeiro (2008). }\end{array}$ & Indicada & Quase experimental, & $\begin{array}{l}3 \text { mães e avó. Grupo semanal (duas vezes } \\
\text { por semana), } 28 \text { horas. Clínica escola. }\end{array}$ \\
\hline $\begin{array}{l}\text { Borges \& Marturano } \\
\text { (2003). }\end{array}$ & Universal & Quase experimental, & $\begin{array}{l}55 \text { crianças. Grupo semanal (duas ou três } \\
\text { vezes por semana), seis meses, } 22 \text { horas. } \\
\text { Escola. }\end{array}$ \\
\hline $\begin{array}{l}\text { Borges \& Marturano } \\
(2009) \text {. }\end{array}$ & Universal & Quase experimental & 61 crianças. Grupo diário, 20 min. Escola. \\
\hline $\begin{array}{l}\text { Borges \& Marturano } \\
(2010) .\end{array}$ & Universal & Quase experimental & 91 crianças. Grupo, 1 ano letivo. Escola. \\
\hline Brino \& Williams (2008). & Universal & Pré-experimental, & $\begin{array}{l}101 \text { professores. Grupo semanal: duas } \\
\text { oficinas (1) } 48 \mathrm{~h} \text { capacitação teórica (2) } 16 \text { h: } \\
\text { prática. Escola. }\end{array}$ \\
\hline $\begin{array}{l}\text { Castro, Melo, \& Silvares } \\
\text { (2003). }\end{array}$ & Indicada & Quase experimental & $\begin{array}{l}38 \text { crianças, pais e professores. Grupo. } \\
\text { Quatro meses para as crianças e pais e oito } \\
\text { meses professores. Escola }\end{array}$ \\
\hline $\begin{array}{l}\text { Cia, Barham, \& Fontaine } \\
(2010) .\end{array}$ & Universal & Quase experimental, & 99 crianças. Grupo semanal. 24 horas. Escola. \\
\hline \multirow[t]{2}{*}{$\begin{array}{l}\text { De Micheli, Fisberg, \& } \\
\text { Formigoni (2004). }\end{array}$} & Seletiva & Experimental & $\begin{array}{l}40 \text { adolescentes. Intervenção individual. } 2 \text { a } 3 \\
\text { minutos. Clínica escola. }\end{array}$ \\
\hline & Indicada & Experimental, & $\begin{array}{l}59 \text { adolescentes. Intervenção individual. } 20 \\
\text { minutos. Clínica escola. }\end{array}$ \\
\hline $\begin{array}{l}\text { De Salvo, Mazzaroto, \& } \\
\text { Lohr (2005). }\end{array}$ & Indicada & Pré-experimental & $\begin{array}{l}9 \text { crianças e } 9 \text { pais. Grupo semanal. } 30 \text { horas. } \\
\text { Escola e clínica escola. }\end{array}$ \\
\hline $\begin{array}{l}\text { Elias \& Marturano } \\
\text { (2005). }\end{array}$ & Indicada & Pré-experimental & $\begin{array}{l}\text { (1) } 17 \text { crianças, grupo semanal, } 40 \text { h, clínica } \\
\text { escola. (2) } 17 \text { mães, grupo quinzenal, } 15 \text { h. } \\
\text { Clínica escola. }\end{array}$ \\
\hline $\begin{array}{l}\text { Gonçalves \& Murta } \\
(2008) .\end{array}$ & Indicada & Pré-experimental & $\begin{array}{l}6 \text { crianças. Grupo semanal. } 30 \text { horas. Clínica } \\
\text { escola. }\end{array}$ \\
\hline
\end{tabular}


Tabela 2

Sistematização dos Programas Preventivos Brasileiros Descritos em Artigos (Continuação)

\begin{tabular}{|c|c|c|c|}
\hline \multirow{6}{*}{$\begin{array}{l}\text { Gomes, Minayo, Assis, } \\
\text { Njaine, \& Schenker } \\
(2007) .\end{array}$} & Universal & \multirow{6}{*}{$\begin{array}{l}\text { Pré-experimental, } \\
\text { descrição das } \\
\text { experiências e } \\
\text { resultados. }\end{array}$} & $\begin{array}{l}\text { Divulgação de informação sobre infância e } \\
\text { adolescência, } 11337 \text { adolescentes. Mídia. }\end{array}$ \\
\hline & Indicada & & $\begin{array}{l}\text { Grupo de dança e percussão. Reforço escolar } \\
\text { (7-13 anos), } 200 \text { sujeitos. Quilombos. }\end{array}$ \\
\hline & Seletiva & & $\begin{array}{l}\text { Escuta e atividades lúdicas, } 500 \text { crianças e } \\
\text { adolescentes. Circo. }\end{array}$ \\
\hline & Indicada & & $\begin{array}{l}\text { Atividades esportivas, palestras e inserção no } \\
\text { mercado de trabalho, } 200 \text { jovens. ONG. }\end{array}$ \\
\hline & Seletiva & & $\begin{array}{l}\text { Capacitação, } 500 \text { funcionários, pais, crianças e } \\
\text { adolescentes. Centro de referência. }\end{array}$ \\
\hline & Seletiva & & Cursos, 950, famílias. Centro Social. \\
\hline \multirow[t]{2}{*}{$\begin{array}{l}\text { Lohr, Pereira, Andrade, } \\
\text { \& Kirchner (2007). }\end{array}$} & \multirow[t]{2}{*}{ Universal } & \multirow[t]{2}{*}{ Pré-experimental } & $\begin{array}{l}4 \text { crianças e } 4 \text { pais. Grupo semanal. } 12 \text { horas, } \\
8 \text { encontros (cada). Escola. }\end{array}$ \\
\hline & & & $\begin{array}{l}\text { Intervenção semanal com } 2 \text { crianças, } 2 \text { pais } \\
\text { com a professora e } 1 \text { professora com os pais. } \\
\text { Escola. }\end{array}$ \\
\hline $\begin{array}{l}\text { Marinho \& Silvares } \\
\text { (2000). }\end{array}$ & Indicada & $\begin{array}{l}\text { Pré-experimental, } \\
\text { follow-up. }\end{array}$ & $\begin{array}{l}28 \text { pais. Intervenções individuais e de grupo, } \\
\text { semanal, } 18 \mathrm{~h}+2 \mathrm{~h} \text { individuais. Clínica } \\
\text { escola. }\end{array}$ \\
\hline Melo \& Silvares (2003). & Indicada & $\begin{array}{l}\text { Experimental, Tipo } \\
\mathrm{AB}\end{array}$ & $\begin{array}{l}\text { (1) } 7 \text { professores, grupo semanal, } 22 \text { horas, } \\
\text { escola. (2) } 13 \text { crianças, grupo semanal, } 39 \\
\text { horas, escola. (3) } 26 \text { pais, grupo quinzenal, } 11 \\
\text { horas. Escola. }\end{array}$ \\
\hline Murta \& Tróccoli (2007). & Universal & Pré-experimental, & $\begin{array}{l}7 \text { adultos (bombeiros de resgate pré- } \\
\text { hospitalar). Grupo semanal, } 13 \text { h30 min. } \\
\text { Corpo de bombeiros. }\end{array}$ \\
\hline
\end{tabular}

Murta \& Tróccoli (2009). Universal Quase experimental, 74 adultos. Grupo semanal, 18 horas, 12 sessões. Universidade.

\begin{tabular}{|c|c|c|c|}
\hline $\begin{array}{l}\text { Murta, Borges, Ribeiro, } \\
\text { Rocha, \& Menezes } \\
(2009) .\end{array}$ & Universal & $\begin{array}{l}\text { Pré-experimental, } \\
\text { Avaliação de } \\
\text { processo e follow-up }\end{array}$ & $\begin{array}{l}\text { (1) } 18 \text { adolescentes, grupo semanal, } 24 \text { h, } 16 \\
\text { sessões, universidade. (2) Grupo de pais, dois } \\
\text { encontros, } 8 \text { h. Universidade. }\end{array}$ \\
\hline $\begin{array}{l}\text { Pinheiro, Haase, Del } \\
\text { Prette, Amarante, \& Del } \\
\text { Prette (2006). }\end{array}$ & Universal & $\begin{array}{l}\text { Pré-experimental, } \\
\text { tipo AB }\end{array}$ & $\begin{array}{l}34 \text { pais, grupo semanal, } 14 \text { h, } 9 \text { encontros. } \\
\text { Escola. }\end{array}$ \\
\hline Prada \& Williams (2007). & Seletiva & $\begin{array}{l}\text { Experimental, tipo } \\
\mathrm{AB}\end{array}$ & $\begin{array}{l}2 \text { monitoras de um abrigo infantil, grupo } \\
\text { semanal, } 18 \text { horas. Abrigo. }\end{array}$ \\
\hline \multirow[t]{2}{*}{$\begin{array}{l}\text { Rodrigues, Dias, \& } \\
\text { Freitas (2010). }\end{array}$} & Indicada & Pré-experimental & $\begin{array}{l}30 \text { crianças. Grupo semanal, } 15 \text { h, } 15 \text { sessões. } \\
\text { Escola. }\end{array}$ \\
\hline & & & $\begin{array}{l}\text { Pais. Grupo mensal. } 4 \text { reuniões de } 50 \text { min. } \\
\text { Escola. }\end{array}$ \\
\hline Silvares \& Melo (2008). & IIndicada & Quase experimental & $\begin{array}{l}240 \text { sujeitos. Grupo } 1 \text { ano letivo. Professores } \\
39 \text { encontros, crianças indicadas: } 22 \text { sessões, } \\
\text { todos os colegas dos alunos e os pais das } \\
\text { crianças indicadas: } 12 \text { sessões. Escola. }\end{array}$ \\
\hline
\end{tabular}


após o término do programa e uma intervenção que apresentou dois follow-ups (3 e 9 meses). Os resultados do follow-up indicaram a manutenção dos ganhos da intervenção ao longo do tempo avaliado. Um estudo sobre a prevenção de álcool e drogas (De Micheli et al., 2004) apresentou diferença significativa da frequência do consumo entre o grupo controle e experimental, indicando a efetividade da intervenção. Entretanto, foi verificada a presença de efeito iatrogênico nessa intervenção, com a redução do consumo de maconha, mas o aumento do consumo de álcool e tabaco. Outro estudo indicou novos ganhos no comportamento de crianças no segundo follow-up, mudanças comportamentais positivas que não haviam sido observadas no pós-teste (Marinho \& Silvares, 2000). Por fim, três dos 25 artigos citaram as políticas públicas, ou seja, $12 \%$ dos estudos incluíram em suas discussões as implicações dos seus resultados para as políticas públicas.

\section{Discussão}

Esta revisão de literatura almejou identificar os centros de pesquisa brasileiros que desenvolvem intervenções preventivas em saúde mental sistematicamente avaliadas e descrever os estudos de avaliação de programas preventivos conduzidos no Brasil. Os resultados apontaram forças, fragilidades e tendências na produção nacional nessa temática. No que diz respeito às forças, há evidências de que a área de prevenção em saúde mental no Brasil tem progredido. O número crescente de artigos ao longo dos anos sugere tal avanço. Isso pode estar associado à produção regular de um conjunto de pesquisadores, conforme demonstram os dados, ainda que esses pesquisadores sejam a menor parte dos que publicam estudos de avaliação de programas preventivos. Para além do crescimento quantitativo, os resultados positivos gerados pelos programas avaliados indicam haver relevância social no campo. Redução em fatores de risco para a saúde mental foi identificada em grande parte dos estudos, o que aponta para a eficácia dos programas desenvolvidos nacionalmente (por exemplo: Borges \& Marturano, 2009; Borges \& Marturano, 2010; Melo \& Silvares, 2003; Marinho \& Silvares, 2000; Murta et al., 2009; Rodrigues, Dias, \& Freitas, 2010; Pinheiro, Haase, Del Prette, Amarante, \& Del Prette, 2006; Prada \& Williams, 2007).

Contudo, a eficácia dos programas analisados nesta revisão deve ser vista com cautela, dadas as limitações metodológicas identificadas no delineamento dos estudos, tais como a escassez de grupos controle, alocação aleatória entre grupos, avaliações de seguimento e avaliações de impacto. Tais características metodológicas constituem a principal fragilidade da área, o que indica haver necessidade de aprimoramentos, com delineamentos que favoreçam a validade interna dos estudos (Melnik \& Atallah, 2011). Portanto, esses resultados evidenciam que delineamentos adequados para estudos de avaliação de eficácia e efetividade de programas de prevenção a fatores de risco ou a transtornos em saúde mental ainda constituem uma necessidade do campo, como apontado há aproximadamente uma década, em revisões prévias (Canoletti \& Soares, 2004; Murta, 2007).

Outras fragilidades foram constatadas, incluindo a ausência de programas preventivos focados em mudanças ambientais complexas, como os dirigidos às organizações e políticas públicas, e programas preventivos dirigidos a adultos em fases tardias do desenvolvimento. A escassez de intervenções para a população adulta e a ausência de intervenções para idosos são preocupantes. As intervenções preventivas poderiam ser conduzidas ao longo do ciclo de vida, visto que transições desenvolvimentais requerem recursos e estratégias de enfrentamento para a adaptação a novas demandas que podem ser significativamente estressoras (Fragelli \& Günther, 2008; Xavier, Ferraz, Marc, Escosteguy, \& Moriguchi, 2003). Poderiam ser realizadas, por exemplo, durante a inserção de jovens no mercado profissional, a gestação do primeiro filho, o ingresso na universidade e a transição para a aposentadoria.

Os resultados sugerem diversas tendências relevantes, incluindo as relativas ao contexto e às características dos estudos desenvolvidos. Quanto ao contexto, os dados indicam que a menor parte dos estudos é apoiada por agências de fomento à pesquisa e que grande parte dos centros de pesquisa se encontra na região sudeste e nenhum deles na região norte do Brasil. Logo, a formação de recursos humanos em nível de pós-graduação e sua alocação em centros de pesquisa de regiões brasileiras menos favorecidas, associada à expansão do fomento para a pesquisa em saúde mental e áreas afins consistem em vias claras de sustentação para o avanço desse campo de pesquisa no Brasil e, por consequência, para a difusão de tecnologias preventivas para a comunidade (Marques, 2012). Quanto às características dos estudos, nota-se a predominância de intervenções em grupo, de longa duração, baseadas no modelo teórico comportamental e cognitivo-comportamental, focadas na promoção de habilidades sociais. A alta frequência de intervenções 
com o conteúdo em habilidades sociais pode estar associada à disponibilidade de instrumentos nacionais com evidências de validade para avaliação de habilidades sociais em crianças e adolescentes (Del Prette \& Del Prette, 2005; Del Prette \& Del Prette, 2009). A disponibilidade desses recursos na literatura nacional pode ser um dos fatores facilitadores da disseminação do conhecimento acerca desse tema e impulsionadores da escolha de intervenções com esse conteúdo.

A análise dos artigos revelou que muitos deles não nomeiam os estudos como preventivos. Isso sugere, uma vez mais, a necessidade de fomentar o ensino de prevenção e, por conseguinte, a identidade do profissional como pesquisador de prevenção. Embora essa inconsistência no uso das terminologias seja respaldada pela literatura (Weisz et al., 2005), visto que os conceitos de prevenção e promoção de saúde apresentam uma forte relação, a não identificação da própria prática como preventiva dificulta a possibilidade da formação de uma rede para colaboração entre pesquisadores brasileiros. Por fim, poucos são os estudos que discutem as implicações de seus resultados para as políticas públicas em saúde mental e áreas afins, tais como promoção de saúde, assistência social, educação, juventude, direitos humanos e gênero. Percebe-se, então, uma lacuna na comunicação entre o pesquisador e os gestores públicos sobre as implicações sociais de seus achados.

Os dados sobre o contexto de aplicação das intervenções levantam a discussão sobre a atuação preventiva do psicólogo nos contextos educacionais, visto que $62,79 \%$ das intervenções foram realizadas em escolas. Esse resultado caminha na direção contrária dos achados da pesquisa nacional sobre a atuação do psicólogo e sua formação (Bastos \& Gondim, 2010). Trata-se de um cenário alarmante, visto que, enquanto os resultados da revisão enfatizam o contexto escolar e o público infanto-juvenil como adequados para receber intervenções preventivas, os dados de atuação profissional apresentam que mais da metade dos psicólogos brasileiros trabalham na clínica tradicional, 53,9\% focados no tratamento dos transtornos mentais e menos de $10 \%$ em contextos educacionais (Bastos \& Gondim, 2010).

\section{Considerações Finais}

Em resumo, essas tendências apontam vários desafios para o avanço da pesquisa em prevenção em saúde mental no Brasil. O aumento de fomento para a pesquisa científica, a expansão dos grupos de pesquisa para regiões menos favorecidas, o ensino de prevenção, o aprimoramento metodológico para avaliação dos estudos e o intercâmbio entre a produção acadêmica e as políticas públicas estão entre as principais necessidades da área. Essas necessidades, se atendidas, podem alicerçar o avanço, ao longo do tempo, dos estudos em prevenção em saúde mental no Brasil.

Este estudo de revisão sistemática apresenta limitações. A primeira limitação é a análise exclusiva das iniciativas de prevenção em saúde mental divulgadas em revistas científicas, priorizadas por profissionais da saúde mental e psicologia. Sugere-se que a análise seja estendida a iniciativas desenvolvidas pelas políticas sociais e em saúde (Sudbrack \& Cestari, 2005), como os programas de governos estaduais e federal, iniciativas de instituições privadas e organizações civis, como ONGs e grupos comunitários, bem como livros, capítulos de livros, a Biblioteca Virtual em Saúde - BVS e outros indexadores. Alguns estudos (cinco no total) não foram analisados em decorrência da limitação de acesso ao periódico e indica-se que novos estudos incluam esses artigos, solicitando os manuscritos aos seus autores brasileiros. Outra limitação é a ausência de análise da qualidade dos estudos. Embora todos os estudos tenham encontrado resultados positivos, o rigor científico na condução de seus delineamentos e métodos não é homogêneo. Sugere-se que estudos futuros analisem a qualidade científica de seus métodos e incluam a avaliação de critérios de eficácia, efetividade e disseminação das intervenções.

Espera-se que o conhecimento das características dos programas preventivos e dos centros de pesquisa que o realizam possam beneficiar gestores públicos e privados e pesquisadores. O benefício direto para os gestores seria oferecer-lhes informações para a tomada de decisão na utilização dos recursos financeiros oriundos de impostos, investindo em programas potencialmente eficazes. Para os pesquisadores, os ganhos poderiam estar relacionados à possibilidade de identificação de seus pares e formalização de parcerias. O trabalho em rede de pesquisas poderá ser um componente importante para manutenção e melhoramento de intervenções preventivas no Brasil e, assim, os pesquisadores poderiam responder ao seu compromisso social de divulgar e transformar o conhecimento em tecnologias úteis e acessíveis à população.

\section{Referências}

Abreu, S. (2012). O estado da arte da pesquisa em prevenção em saúde mental no Brasil: uma revisão sistemática 
(Dissertação de mestrado não publicada). Instituto de Psicologia da Universidade de Brasília, Brasília, Brasil.

Albee, G. W. (1982). Preventing psychopathology and promoting human potential. American Psychologist, 37(9), 1043-1050. Recuperado de http://www. ncbi.nlm.nih.gov/pubmed/7149433

Aquino, T. A. A., Silva, J. P., Figueiredo, A. T. B., Dourado, E. T. S., \& Farias, E. C. S. (2011). Avaliação de uma proposta de prevenção do vazio existencial com adolescentes. Psicologia: Ciência e Profissão, 31(1), 146-159. doi: 10.1590/S1414-98932011000100013

Associação Americana de Psiquiatria (2003). DSM-IV-TR. Manual Diagnóstico e Estatístico dos Transtornos Mentais. ( $4^{a}$ ed.) Porto Alegre: Artmed.

Bastos, A. V. B., \& Gondim, S. M. G. (2010). O trabalho do psicólogo no Brasil. (1 ${ }^{\mathrm{a}} \mathrm{ed}$.) Porto Alegre: Artmed.

Benetti, S. P. C., Ramires, V. R. R. Schneider, A. C., Rodrigues, A. P. G., \& Tremarin, D. (2007). Adolescência e saúde mental: Revisão de artigos brasileiros publicados em periódicos nacionais. $C a$ dernos de Saúde Pública, 23, 1273-1282. doi: 10.1590/ S0102-311X2007000600003

Bolsoni-Silva, A. T., \& Marturano, E. M. (2010). Evaluation of group intervention for mother/caretakers of kindergarten children with externalizing behavioural problems. Interamerican Journal of Psychology, 44(3), 415-421. Recuperado de http://www.redalyc.org/articulo.oa?id $=28420658002$

Bolsoni-Silva, A. T., Silveira, F. F., \& Marturano, E. M. (2008). Provendo habilidades sociais educativas parentais na prevenção de problemas de comportamento. Revista de Terapia Comportamental Cognitiva, 10(2), 125-142. Recuperado de http://www.usp. $\mathrm{br} / \mathrm{rbtcc} /$ index.php/RBTCC/article/view/182

Bolsoni-Silva, A. T., Silveira, F. F., \& Ribeiro, D. C. (2008). Avaliação dos efeitos de uma intervenção com mães/cuidadores: Contribuições do treinamento de habilidades sociais. Contextos Clínicos, 1(1), 19-27. Recuperado de http://pepsic.bvsalud. org/pdf/cclin/v1n1/v1n1a03.pdf

Borges, D. S. C., \& Marturano, E. M. (2003). Desenvolvendo habilidades de solução de problemas interpessoais no ensino fundamental. Paidéia, 12(24), 185-193. Recuperado de http://www.scielo.br/pdf/paideia/v12n24/07.pdf
Borges, D. S. C., \& Marturano, E. M. (2009). Aprendendo a gerenciar conflitos: Um programa de intervenção para a $1^{a}$ série do ensino fundamental. Paidéia, 19(42), 17-26. doi: 10.1590/ S0103-863X2009000100004

Borges, D. S. C., \& Marturano, E. M. (2010). Melhorando a convivência em sala de aula: responsabilidades compartilhadas. Temas em Psicologia, 18(1), 123-136. Recuperado de http://pepsic.bvsalud.org/pdf/ tp/v18n1/v18n1a11.pdf

Brino, R. F., \& Williams, L. C. A. (2008). Professores como agentes de prevenção do abuso sexual infantil. Educação \& Realidade, 33(2), 209-230. Recuperado de http://seer.ufrgs.br/educacaoerealidade/ article/viewFile/7073/4389

Campbell, D. T., \& Stanley, J. C. (1979). Delineamentos experimentais e quase experimentais de pesquisa. ( $3^{\mathrm{a}} \mathrm{ed}$.) São Paulo: EPU.

Canoletti, B., \& Soares, C. B. (2004). Programas de prevenção ao consumo de drogas no Brasil: Uma análise da produção científica de 1991 a 2001. Interface - Comunicação, Saúde e Educação, 16, 115-129. Recuperado de http://www.scielo.br/pdf/icse/ v9n16/v9n16a10

Castro, R. E. F., Melo, M. H. S., \& Silvares, E. F. M. (2003). O julgamento de pares de crianças com dificuldades interativas após um modelo ampliado de intervenção. Psicologia: Reflexão e Crítica, 16(2), 309318. Recuperado de http://www.scielo.br/pdf/ prc/v16n2/a11v16n2.pdf

Cia, F. Barham, E. J., \& Fontaine, A. M. G. V. (2010). Impacto de uma intervenção com pais: $\mathrm{O}$ desempenho acadêmico e comportamento das crianças na escola. Psicologia: Reflexão e Crítica, 23(3), 533543. doi: 10.1590/S0102-79722010000300014

Conselho Nacional de Desenvolvimento Científico e Tecnológico (2012). Ajustes na Plataforma Lattes estimulam a divulgação científica. Recuperado de http://www.ensp.fiocruz.br/portal-ensp/ informe/site/materia/detalhe/29770

Dalton, J. H., Elias, M. J., \& Wandersman, A. (2007). Community psychology: Linking individuals and communities. ( $2^{\mathrm{a}}$ ed.). Belmont, California: Thomsom Wadsworth.

Del Prette, Z. A. P., \& Del Prette, A. (2005). Sistema Multimídia de Habilidades Sociais para Crianças. São Paulo: Casa do Psicólogo. 
Del Prette, Z. A. P., \& Del Prette, A. (2009). IHSA Inventário de Habilidades Sociais para Adolescentes. São Paulo: Casa do Psicólogo.

De Micheli, D., Fisberg, M., \& Formigoni, M. L. O. S. (2004). Estudo da efetividade da intervenção breve para o uso de álcool e outras drogas em adolescentes atendidos num serviço de assistência primária à saúde. Revista da Associação Médica Brasileira, 50(3), 305-313. doi: 10.1590/S0104-42302004000300040

De Salvo, C. G, Mazzaroto, I. H. K., Lohr, S. S. (2005). Promoção de habilidade sociais em pré-escolares. Revista Brasileira de Crescimento e Desenvolvimento Humano, 15(1), 46-55. Recuperado de http://pepsic. bvsalud.org/pdf/rbcdh/v15n1/06.pdf

Doughty, C. (2005). The effectiveness of mental health promotion, prevention and early intervention in children, adolescents and adults: A critical appraisal of the literature. New Zealand Health Technology Assessment Report, 8(2), 7-12. Recuperado de http://www.crd.york.ac.uk/crdweb/ShowRecord. asp?ID $=32005000403$

Durlak, J. A., Wells, A. M. (1997). Primary prevention mental health programs for children and adolescents: A meta-analytic review. Americam Journal of Community Psychology, 25(2), 115-142. Recuperado de http://www.ncbi.nlm.nih.gov/pubmed/9226860

Eddy, J. M. (2011). One day: In search of training opportunities in prevention science. SPR Community, 1(1), 5-6. Recuperado de www.preventionresearch.org

Elias, L. C., \& Marturano, E. M. (2005). Oficinas de linguagem: Proposta de atendimento psicopedagógico para crianças com queixas escolares. Estudos de Psicologia, 10(1), 53-61. doi: 10.1590/ S1413-294X2005000100007

Flay, B. R., Biglan, A., Boruch, R. F, Castro, F. G., Gottfredson, D., Kellan, S., Mosciki, E. K., Schinke, S., Valentine, J. C., \& Ji, P. (2005). Standards of evidence: Criteria for efficacy, effectiveness and dissemination. Prevention Science, 6(3), 151-173. Recuperado de http://www.ncbi.nlm.nih.gov/ pubmed/16365954

Fragelli, T. B. O., \& Günther, I. A. (2008). A promoção da saúde na perspectiva social ecológica. Revista Brasileira em Promoção da Saúde, 21(2), 151-158. doi:10.5020/18061230.2008.p151
Gomes, R., Minayo, M. C. S., Assis, S. G., Njaine, K., \& Schenker, M. (2006). Êxitos e limites na prevenção da violência: Estudo de caso de nove experiências brasileiras. Ciência e Saúde Coletiva, 11 (suppl 0), 1291-1302. Recuperado de http://www.arca.fiocruz.br/handle/icict/202

Gonçalves, E. S., \& Murta, S. G. (2008). Avaliação dos efeitos de uma modalidade de treinamento de habilidades sociais para crianças. Psicologia: Reflexão e Crítica, 21(3), 430-436. doi: 10.1590/ S0102-79722008000300011

Kazdin, A. E. (1982). Methods for clinical and applied settings. ( $2^{\mathrm{a}}$ ed.). New York: Oxford University Press.

Lohr, S. S., Pereira, A. C. S., Andrade, A. L. M., \& Kirchner, L. F. (2007). Avaliação de programas preventivos: Relato de experiência. Psicologia em Estudo, 12(3), 641-649. doi: 10.1590/ S1413-73722007000300022

Marinho, M. L., \& Silvares, E. F. M. (2000). Evaluación de la eficácia de um programa de entrenamiento de padres em grupo. Psicologia Conductual, 8(2), 299-318. Recuperado de http://www.psicologiaconductual. com/PDFespanol/2000/art07.2.08.pdf

Marques, F. (2012). Muito além das patentes. Pesquisa FAPESP, 197, 20-27. Recuperado de http:// revistapesquisa.fapesp.br/2012/07/16/ muito-alem-das-patentes/

Melnik, T., \& Atallah, A. N. (2011). Psicologia baseada em evidências: Articulação entre a pesquisa e prática clínica. Em T. Menik \& A. N. Atallah (Eds.), Psicologia Baseada em Evidências: Provas Científicas da Efetividade da Psicoterapia (pp. 3-13). São Paulo: Santos.

Melo, M. H. S., \& Silvares, F. M. (2003). Grupo cognitivo-comportamental com famílias de crianças com déficits em habilidades sociais e acadêmicas. Temas em Psicologia, 11(2), 122-133. Recuperado de http://pepsic.bvsalud.org/pdf/tp/v11n2/ v11n2a06.pdf

Muñoz, R. E., Mrazek, P. J., \& Haggerty, R. J. (1996). Institute of medicine report on prevention of mental disorders. American Psychologist, 51(11), 1116-1122. Recuperado de http://www.ncbi.nlm.nih.gov/ pubmed/8937259

Murta, S. G., Borges, F. A., Ribeiro,... D. C., Rocha, E. P., Menezes, J. C. L. (2009). Prevenção primária em saúde na adolescência: Avaliação de um programa 
de habilidades de vida. Estudos em Psicologia, 14(3), 181-189. doi: 10.1590/S1413-294X2009000300001

Murta, S. G., \& Trócoli, B. T. (2007). Stress ocupacional em bombeiros: Efeitos de intervenção baseada em avaliação de necessidades. Estudos de Psicologia, 24(1), 41-51. Recuperado de http://www.scielo. br/pdf/estpsi/v24n1/v24n1a05.pdf

Murta, S. G., \& Trócoli, B. T. (2009). Intervenções psicoeducativas para manejo de estresse ocupacional: Um estudo comparativo. Revista Brasileira de Terapia Comportamental Cognitiva, 11(1), 25-42. Recuperado de http://www.usp.br/rbtcc/index.php/RBTCC/ article/view/382

Murta, S. G. (2011) Aproximando ciência e comunidade: difusão de programas de habilidades sociais baseados em evidências. In: Del Prette, A., \& Del Prette, Z. A. P. (Orgs.) Habilidades sociais: intervencões efetivas em grupo (pp. 83-114). São Paulo: Casa do Psicólogo.

O'Connel, M. E., Boat, T., \& Warner, K. E. (2009). Preventing mental, emotional, and behavioral disorders among young people: Progress and possibilities. Washington, DC: The National Academies Press.

Pinheiro, M. I. S., Haase, V. G., Del Prette, A., Amarante, C. L. D., \& Del Prette, Z. A. P. (2006). Treinamento de habilidades sociais educativas para pais de crianças com problemas de comportamento. Psicologia: Reflexão e Crítica, 19(3), 407-414. doi: 10.1590/S0102-79722006000300009

Prada, C, G., Williams, L. C. A., \& Weber, L. N. D. (2007). Abrigos para crianças vítimas de violência doméstica: Funcionamento relatado pelas crianças e pelos dirigentes. Psicologia: Teoria e Prática, 9(2), 14-25. Recuperado de http://www.nac.ufpr.br/ artigos_do_site/2007_Abrigos_para_criancas_vitimas_de_violencia_domestica.pdf
Rodrigues, M. C., Dias, J. P., \& Freitas, M. F. R. L. (2010). Resolução de problemas interpessoais: Promovendo o desenvolvimento sociocognitivo na escola. Psicologia em Estudo, 15(4), 831-839. Recuperado de http://www.redalyc.org/articulo. oa?id $=287123084019$

Silvares, E. F. M., \& Melo, M. H. S. (2008). Evolução do manejo clínico dos problemas de conduta: Do tratamento à prevenção. Revista Psicolog, 1(1), 4-20.

Sudbrack, M. F. O., \& Cestari, D. M. (2005). O modelo sistêmico e da educação para a saúde na prevenção da drogadição no contexto da escola: Propostas de projeto piloto SENAD/ MEC e UnB. Em Simpósio Internacional do Adolescente. Anais do $1^{\circ}$ Simpósio Internacional do Adolescente. São Paulo, São Paulo, Brasil.

Weisz, J. R., Sandler, I. N., Durlak, J. A., \& Anton, B. S. (2005). Promoting and protecting youth mental health through evidence-based prevention and treatment. American Psychologist, 60(6), 628-648. Recuperado de http://www.ncbi.nlm.nih.gov/ pubmed/16173895

World Health Organization (2004). Prevention of mental disorders - effective interventions and policy options. Paris, França. Retirado de: http:// www.who.int/mental_health/evidence/en/ prevention_of_mental_disorders_sr.pdf

Xavier, F. M. F., Ferraz, M. P. T., Marc, N., Escosteguy, N. V., \& Moriguchi, E. H. (2003). Eldery people's definition of quality of life. Revista Brasileira de Psiquiatria, 25(1), 31-39. doi: 10.1590/ S1516-44462003000100007

Recebido em: 26/11/2014

Primeira reformulação em: 13/03/2015 Aprovado em: 09/04/2015 
Nota dos autores:

Esta pesquisa recebeu o apoio do CNPq - Conselho Nacional de Desenvolvimento Científico e Tecnológico.

Sobre as autoras:

Samia Abreu é mestre pelo Programa de Psicologia Clínica e Cultura da Universidade de Brasília, atua como supervisora nacional de programas preventivas do Ministério da Saúde, sendo responsável pela adaptação, implantação e disseminação das intervenções preventiva e como sócia da FLUME Consultoria, e membro do Grupo de Estudos em Prevenção e Promoção de Saúde no Ciclo de Vida (GEPPSVida - UnB).

E-mail:abreu.samia@gmail.com

Ana Aparecida Vilela Miranda é mestranda do Departamento de Psicologia Clínica e Cultura pela Universidade de Brasília, psicóloga, formada pela Universidade de Brasília, pesquisadora do Grupo de Estudos em Prevenção e Promoção da Saúde no Ciclo da Vida (GEPPS-Vida) e nas áreas de Prevenção, Políticas Públicas, desenvolvimento humano, e promoção em saúde mental.

E-mail:aavmiranda@gmail.com

Sheila Giardini Murta é professora adjunta no Departamento de Psicologia Clínica, orientadora no Programa de Pós-Graduação em Psicologia Clínica e Cultura da Universidade de Brasília, pós-doutora pela Universidade Federal de São Carlos e Universidade de Maastricht-Holanda, membro do GT-ANPEPP - Relações Interpessoais e Competência Social e coordenadora do Grupo de Estudos em Prevenção e Promoção de Saúde no Ciclo de Vida (GEPPSVida - UnB).

E-mail: giardini@unb.br

Contato com as autoras:

Samia Abreu

Departamento de Psicologia Clínica, Instituto de Psicologia, Universidade de Brasília, Campus Darcy Ribeiro, Brasília, DF

CEP: 70910-900 
